\title{
LES RELIGIONS DANS L'ESPACE PUBLIC EN FRANCE, ENTRE HIER ET AUJOURD'HUI ?
}

En France et ailleurs, on peut remarquer combien -au-delà des résistances- la place des religions dans l'espace public est en plein bouleversement, combien est chahutée la part traditionnelle réservée aux cultes

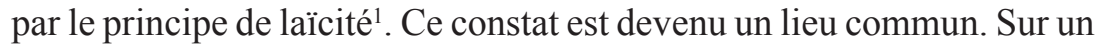
arrière-fond fait de déconstructions et de mondialisation, on l'explique couramment par la rencontre difficile des politiques de sécularisation et des mouvements d'immigration. Face aux changements perçus comme des menaces pour l'ordre public, il semble alors nécessaire de revisiter les notions très embrouillées qui servent à définir -depuis la loi du 9

* Professeur agrégé des Facultés de droit ; Membre du Centre Toulousain d'Histoire du Droit et des Idées Politiques ; Université Toulouse 1 Capitole ; 2, rue du Doyen-Gabriel-Marty, 31042 Toulouse cedex 09 ; République française ; email : Christine.menges-le-pape@ ut-capitole.fr.

${ }^{1}$ Liberté d'expression et liberté de communication, Les nouveaux cahiers du Conseil constitutionnel, Paris, éd. Dalloz, 2012, n³6 ; Laïcité et liberté religieuse. Recueil de textes et de jurisprudence, Paris, Les éditions des journaux officiels, 2011 ; La laïcité, archives de philosophie du droit, Paris, éd Dalloz, mars 2005, t. 48 ; Le pluralisme, archives de philosophie du droit, Paris, éd Dalloz, mars 2006, t. 49 ; Philippe Chiappini, Le droit et le sacré, Paris, éd. Dalloz, 2006 ; Claude Durand-Prinborgne, La laïcité, éd. Dalloz, 2004 ; Dominique Laszlo-Fenouillet, La conscience, Paris, LGDJ, 1993 ; Convictions philosophiques et religieuses et droits positifs, colloque national de Moncton, Bruxelles, éd. Bruylant, 2010. 
décembre 1905- les territoires dévolus aux religions devenus des aires mouvantes. Or cette recherche exige que soient confrontées les situations historiques entre hier et aujourd'hui, d'abord parce que le passé religieux et politique contient de pareilles mutations ; ensuite parce que les comparaisons informent beaucoup sur ce qui parait nouveau bien que lié à des spéculations entamées auparavant lors des grandes ruptures de la conscience occidentale prolongées jusqu'à nous. Dans ce jeu de reflets entre hier et aujourd'hui, peuvent être saisies les différences essentielles entre les cultes reconnus en 1905 et les religions géographiquement ou historiquement nouvelles que l'État protège et qui -à cause de leur conception théocratique- ne peuvent supporter les mêmes régulations, car elles ne sont pas toutes fondées sur le principe de distinction du profane et du sacré. Comme l'explique le philosophe Rémi Brague dans la préface du livre Dieu des chrétiens, Dieu des musulmans ${ }^{2}$, la méthode comparative permet de prévenir les errements destructeurs liés aux fausses ressemblances qui empêchent de distinguer les religions et de respecter l'autre.

$\mathrm{Au}$ milieu de ces temps livrés paradoxalement à la pluralité spirituelle et à son refus, les notions à interroger sont dispersées dans plusieurs formules, et leur éparpillement signale déjà les embarras d'un vocabulaire qui tente d'exprimer la distinction difficile du spirituel et du temporel et de dire les parts respectives. Il s'agit des mots souvent répétés depuis l'Antiquité d'espace public ou privé. Aristote avait déjà établi la fracture entre $\pi$ ó $\lambda$ is et oíkos. Ces termes contiennent pour la France une dimension complexe à la fois juridique et d'histoire territoriale, avec la loi municipale du 5 avril 1884. Ces mots furent surtout repris pour parler des libertés religieuses et politiques à la suite des travaux très connus de Jürgen Habermas, L'espace public : archéologie de la publicité . D'ailleurs, dans Foi et savoir ${ }^{4}$, Habermas remarque ce mouvement qui suscite de nombreux colloques et qui va vers « la

\footnotetext{
${ }^{2}$ François Jourdan, Dieu des chrétiens, Dieu des musulmans, Paris, éditions de l'CEuvre, 2008.

${ }^{3}$ Jürgen Habermas, L'espace public : archéologie de la publicité, Paris, Payot, rééd. 1988.

${ }^{4}$ Jürgen Habermas, Foi et Savoir, L'avenir de la nature humaine. Vers un eugénisme libéral ?, Paris, Gallimard, 2002, p. 151.
} 
persistance des communautés religieuses dans un environnement qui continue à se séculariser ». Il s'agit ici de la confrontation toujours de plus en plus compliquée entre le pluralisme religieux et la laïcité dans la diversité des espaces. Lors du colloque Le contrôle des religions organisé par l'Institut catholique de Toulouse en septembre 2014, Jean-Louis Bianco, directeur de l'Observatoire de la laïcité, distingue même un troisième espace, l'espace commun qui s'ajoute aux espaces public et privé. Or cette distinction a pu surprendre par la difficulté de la définition juridique de l'espace commun ${ }^{5}$.

Pour présenter ces territoires désormais en plein mouvement, il $\mathrm{y}$ a aussi les mots -à tonalité politique et spirituelle- de place et de forum avec le très significatif dérivé canonique de for ; ceux de vie publique ou privée plutôt utilisés par les rédacteurs des codifications $\mathrm{du}$ premier $\mathrm{XIX}^{\mathrm{e}}$ siècle et par la doctrine des débuts du $\mathrm{XX}^{\mathrm{e}}$ siècle. Toujours pour signifier les distinctions juridiques entre public et privé, il y a les notions de conscience et de liberté de conscience, elles relèvent des droits subjectifs et de l'espace privé. Mais la liberté de conscience s'ouvre sur un exercice nécessairement public des libertés d'expression et de cultes, même si l'on conçoit de plus en plus les lieux de cultes comme des espaces clos et donc privés. Viennent enfin les termes d'un usage plus moderne et peut-être à la fois doctrinal et médiatique, ceux de sphère publique ou privée. Ils possèdent des résonnances philosophiques et quasi-ésotériques issues de la Renaissance, avec la vision homéomorphe de la sphère. C'est l'idée de la coincidentia oppositorum, que contient tellement cette figure géométrique ${ }^{6}$. La théorie fut très à la mode dès le $X V^{\mathrm{e}}$ siècle, avec Nicolas de Cues, dans un contexte dominé par la prise de Constantinople ; on a pu l'utiliser à travers des politiques imprégnées d'ésotérisme pour dépasser les oppositions des religions et donner un nouveau sens à la tolérance. Les polygones -sous

${ }^{5}$ Jean-Louis Bianco, « Discours introductif, colloque international Le contrôle des religions, institut catholique de Toulouse, septembre 2014 : «L'espace privé : espace où l'on est totalement libre, sous la seule réserve du respect de la loi. L'espace public : espace de l'État, des collectivités locales, des services publics (bâtiments et locaux publics, etc.), des établissements scolaires. L'espace commun : espace commun à tous, comme la rue par exemple, à ne pas confondre avec l'espace public au sens précédent ».

${ }^{6}$ Pierre Béhar, Les langues occultes de la Renaissance, Paris, Desjonquères, 1996. 
l'effet de pressions séculières et par l'exemple- devaient devenir des sphères, c'est-à-dire des non-polygones. Il s'agit de déjà proposer une fusion des identités.

Ce vocabulaire qui spatialise ce face à face entre le sacré et le profane est un tempérament surtout français. Ailleurs, en Espagne et en Italie, surtout en Angleterre, on parle des opinions religieuses ou politiques sans les implanter sur un territoire, sans les intégrer, ni les délimiter. Cette particularité française peut renseigner sur quelques finalités de la laïcité, parfois partagées entre volonté d'accueil ou de rejet des religions, selon les orientations ministérielles.

Or tous ces termes qui disent ainsi les territoires politiques et religieux déterminés par la laïcité française, sont affectés par l'instabilité. Ils sont devenus désormais polysémiques. Chacun met autre chose derrière ces mots. Ainsi ces expressions sont-elles encombrées par l'équivoque du relativisme, elles sont surtout marquées par cette tendance à la simplification qui depuis longtemps veut rendre équivalente la sphère politique à la sphère publique, la sphère religieuse à la sphère privée. Par ces équations rapides, la sphère religieuse doit être « mise à part» pour reprendre le Dictionnaire pédagogique et d'instruction primaire de Ferdinand Buisson que l'on redécouvre aujourd'hui par le biais de ses nouvelles éditions ${ }^{7}$ et par les écrits sur la morale républicaine du ministre Vincent Peillon ${ }^{8}$. Toutefois cette équivalence devenue courante est une ancienne et récurrente hésitation culturelle, fort utile lorsque les situations se brouillent, lorsque les mots n'ont plus de véritable sens et qu'ils ont perdu -même pour les juristes- de leur solidité à cause de l'inflation verbale du droit et de l'hyper-relativisme introduit dans le style du siècle. Il s'agit aussi d'un risque de négation culturelle, avec cette interrogation qui surgit toujours en pleine crise : peut-on interdire l'espace public aux religions, par peur d'une contestation du pouvoir? Alors que l'inverse est plus difficile : en Occident

${ }^{7}$ Ferdinand Buisson, Dictionnaire pédagogique et d'instruction primaire, Paris, 1887. Il y eut une autre édition en 1911. À partir de 2004, la Bibliothèque nationale de France, sur Gallica, proposa une version numérisée de ces éditions.

${ }^{8}$ Vincent Peillon, Une religion pour la république, la foi lä̈que de Ferdinand Buisson, Paris, Éditions du Seuil, 2010. 
l'autorité religieuse n'a jamais pu empêcher l'émergence d'espaces publics. Peut-on taire la part du sacré, même si la modernité tente de le démontrer à travers la techno-science et le matérialisme, même si prévaut cette perspective ouverte par la III ${ }^{e}$ République française de limiter à elle-même l'espace public ? Pour dire cette confusion entre religion et sphère privée, il y a ici le souvenir -très enseigné depuis le $\mathrm{XIX}^{\mathrm{e}}$ siècle- des gauchissements de l'augustinisme politique qui s'établit avec la fin bousculée du bas Empire et de ses régressions culturelles. On pose alors les assimilations entre la cité de Dieu et l'Église, puis entre la cité des hommes et l'État. Henri-Irénée Marrou parle -pour cette réduction- d'une sous-mouture d'Augustin'. Or l'expression qui signale un appauvrissement, s'applique aux enfermements actuels des religions dans la sphère privée, celle de l'intimité des consciences, celle de l'espace de plus en plus étroit des cultes. N'est-ce pas là le signe d'une opinion qui ne saisit plus la nature publique des religions, c'est-àdire leur participation à la res publica par l'éducation et la charité. C'est ici ignorer la part multiséculaire des religions. C'est réduire le sens de « mettre à part », le rapprocher d'un confinement dans le seul espace privé. Cette incompréhension montre l'ébranlement d'une société qui forge sa pensée dans l'oubli du socle classique des connaissances et surtout dans la peur de nouvelles altérités, comme s'il était impossible de penser plusieurs espaces publics, de parler d'espaces publics au pluriel comme l'histoire l'a souvent fait depuis l'Antiquité entre l'acropole et l'agora.

Dès lors le manque de stabilité des termes, conjugué aux simplifications qui en découlent, crée des alarmes avec des interventions contradictoires et des malentendus qui rendent difficile un dialogue respectueux et normal entre religions, et surtout entre États et religions. Ces mouvements sont fréquents dans les périodes de forte cassure. Ils provoquent une opinion qui s'installe dans le mélange des domaines et qui pense rapidement, à travers l'intuition des émotions, selon ceux qui seuls ont le droit de parler et pourtant qui sont « incapables de penser » pour reprendre les mots d'Arlette Farge dans Dire et mal dire, car ils

${ }^{9}$ Henri-Irénée Marrou, Décadence romaine ou antiquité tardive ?, Paris, 1977 ; Saint Augustin et l'augustinisme, Paris, 2003. 
croient que penser signifie seulement penser par soi-même et au gré des événements récents, dans l'oubli du savoir historique ${ }^{10}$.

Or par cette volonté moderne de cloisonner les religions dans la sphère privée, les autonomies et la nature publique du politique et du religieux sont mises à mal, dans une méconnaissance idéologique du principe de distinction et de son histoire, ce principe qu'il faut étudier à travers les réalités passées et actuelles, celles les politiques d'intégration, celles des diversités religieuses.

\section{L'ESPACE PUBLIC ET LES MOUVEMENTS DES POLITIQUES D'INTÉGRATION : ENTRE HIER ET AUJOURD'HUI}

En France, depuis la loi de séparation des Églises et de l'État, l'espace réservé aux religions est défini à partir du concept de laïcité, nous le savons. Or à cause du nomadisme spirituel que connaît l'Occident et des désordres introduits dans les notions d'espace public et cultuel, la définition de la laïcité est de plus en plus difficile à rendre. Pourtant c'est cette notion qui est le socle de la séparation. Aujourd'hui, la doctrine et la jurisprudence ont inclus la laïcité parmi les principes fondamentaux de la République. C'est désormais un principe à valeur constitutionnelle. " La France est une république indivisible, laïque, démocratique et sociale ", tel est l'article $1^{\text {er }}$ de la Constitution du 4 octobre 1958. Tel est le fondement de la jurisprudence du Conseil constitutionnel. C'est aussi l'avis de Jean-Louis Bianco : « la laïcité n'est pas une simple opinion, elle est devenue un principe constitutionnel partagé par tous $\gg{ }^{11}$.

Mais au-delà de la reconnaissance d'un principe juridique, se posent toujours les incertitudes de ses origines et de sa définition

${ }^{10}$ Arlette Farge, Dire et mal dire, l'opinion publique au XVIII' siècle, Paris, Seuil, 1992.

${ }^{11}$ Discours de Jean-Louis Bianco, 17 février 2014, « Réponse aux propos du 10 février 2014 publiés sur le site Internet de l'UFAL concernant les travaux de l'Observatoire de la laïcité » : «En effet, en France, la laïcité s'inscrit dès son origine comme un principe supérieur et non comme une simple opinion. La laïcité est devenue un principe constitutionnel d'organisation de l'État. Si la laïcité était une simple «tendance », elle serait dévaluée et réduite à un choix qui ne serait plus le principe constitutionnel partagé par tous ». 
qui ont suscité un déferlement de doctrine et d'ouvrages. Dans le Dictionnaire pédagogique, à l'article « laïque », Ferdinand Buisson se risque dans l'étymologie du terme pour justifier d'emblée la séparation du clergé. Il expose que « ce mot lä̈que fut employé dans les premières communautés chrétiennes, où l'on parlait grec, il servit à désigner ceux qui n'étaient pas du clergé $\rangle^{12}$. Or cette dissociation n'est pas nouvelle, elle appartient à la tradition occidentale, elle relève de la distinction du spirituel et du temporel. De nombreuses références bibliques disent cette façon d'agir du Dieu de la Bible qui procède par séparation de la lumière et des ténèbres, des eaux et du firmament, du jour et de la nuit. Pour l'espace public, c'est le « rendez à César ce qui est César », avec cette distinction faite entre l'Empire et l'Église naissante, pour empêcher la fusion et permettre la réciprocité. Très tôt on retrouve cette répartition avec Ambroise de Milan, Augustin d'Hippone ; puis au $\mathrm{VI}^{\mathrm{e}}$ siècle, avec le «duo sunt » prononcé par le pape Gélase I Ir13 ; en 793, c'est Alcuin le conseiller et ami de Charlemagne qui écrit « le pouvoir séculier et le pouvoir spirituel sont séparés $»^{14}$. Puis il y aura la théorie des deux glaives qui maintient plus difficilement cette partition. Dès le $X V^{e}$ siècle, avec les crises de la conscience européenne, se rouvre le conflit entre le prince et la religion, conflit qui auparavant avait été apaisé par l'accord politico-religieux du Moyen Âge dans une tripartition des ordres où chacun semblait pouvoir trouver sa place. La querelle durera jusqu'à la fin du $\mathrm{XIX}^{\mathrm{e}}$ siècle, elle conduit à une primauté du temporel. Ferdinand Buisson reprendra l'idée de séparation pour préciser « les clercs, c'est une fraction de la société qui se tient pour mise à part $\rangle^{15}$. Or, ici le sens des termes a changé. La séparation n'est plus conçue pour établir un dialogue, mais plutôt pour afficher un écartement. Elle sert à isoler la part spirituelle.

Viennent ensuite les aspects démocratiques de la laïcité interrogés par le Dictionnaire pédagogique, ils contiennent une opaci-

${ }^{12}$ Ferdinand Buisson, Dictionnaire pédagogique et d'instruction primaire, op. cit., article « laïque ».

${ }^{13}$ Gelasius, De anthemis vinculo, Migne JP, Patrologia latina, t. 59, p. 108.

${ }^{14}$ Alcuin, Epistolae, Berlin, Ernst Dümmler, 1895, t. 4, p. 48.

${ }^{15}$ Ferdinand Buisson, Dictionnaire pédagogique et d'instruction primaire, op. cit., article « laïque ». 
té que comporte la question alors posée dans 1'article « laïque »: "Quelle est 1'origine et la signification exacte de ce mot " laïque » d'où la génération contemporaine a tiré le néologisme laïcité ? ${ }^{16}$. Pour dire curieusement le caractère démocratique, Ferdinand Buisson se sert de l'utilisation médiévale et religieuse du mot : « Il y avait dans les couvents des frères lais, des sœurs laies, c'étaient des personnes qui vivaient dans l'enceinte d'une communauté monastique sans avoir prononcé de vœux »; " cette forme représente, est-il expliqué, le latin laïcus ». Sont ensuite montrées les origines grecques, c'est-à-dire l'adjectif $\lambda \alpha i ̈ \kappa o ́ \varsigma$, dérivé du substantif $\lambda \alpha$ ó $\varsigma$ qui signifie « peuple ». De cette racine grecque, Ferdinand Buisson en déduit par l'ambiguïté d'un raccourci étymologique que «le véritable sens, le sens primitif du mot laïque est donc celui de populaire $\gg{ }^{17}$. Vient ainsi la vision politique des laïques, « c'est le peuple, c'est la masse non mise à part, c'est tout le monde, c'est l'esprit démocratique et populaire $\|^{18}$. Or les explications contenues dans cet article « laïque » dérivent d'une synonymie rapide et floue, peut-être idéologique, entre laïque et démocratique, contiguïté que l'Antiquité n'établissait pas. Le $\lambda \alpha o ́ s$, ce n'est pas le $\delta \tilde{\eta} \mu$ o $_{\text {qui }}$ recouvre le peuple comme communauté politique. Le mot démocratique -employé par le Dictionnaire pédagogique-vient de $\delta \tilde{\eta} \mu \mathrm{o}$, et non de $\lambda \alpha$ ós. L'un est politique, l'autre d'utilisation religieuse. C'est le $\lambda \alpha o ́ \varsigma$

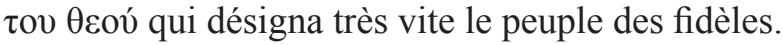

Et la distinction est importante, car dès la fin du XVIII ${ }^{\mathrm{e}}$ siècle, le mot laïque est alors utilisé par les orateurs de la Révolution dans un sens mystique, mais anti-catholique, pour soutenir une spiritualité républicaine, c'est-à-dire un culte politique. Ici il y a les illustrations de la Constitution civile du clergé, puis le culte de l'Être suprême et les martyrs révolutionnaires. Toutefois en 1789 , cette spiritualité politique n'est pas neuve. Elle est préparée auparavant dans le royaume par la Renaissance et la réception d'une philosophie hermétique venue de l'Antiquité païenne qui confond le temporel au spirituel sous l'autorité du prince. Les origines de cette confessionnalisation des États sont donc

\footnotetext{
${ }^{16}$ Ibidem.

${ }^{17}$ Ibidem.

${ }^{18}$ Ibidem.
} 
hellénistiques, on les retrouve ensuite à Rome avec le culte impérial. Puis c'est l'application de la formule cujus regio ejus religio, prônée par la Réforme, souhaitée par Catherine de Médicis, d'abord pratiquée par l'Angleterre et les principautés allemandes lors des millésimes 1560-1570, mais que refusent les ligueurs français et les ultramontains. En France, la religion ne pouvait être fille du prince. La conversion de Henri IV en est une démonstration éclatante. Or par la suite, le gallicanisme politique l'emporte. Sûrement dans un souci irénique très enchevêtré, la religion se fait fille du Très Chrétien, ce qui dirige vers un jus circa sacra -un droit du Prince sur le sacré- vers une spiritualité politique qui n'est pas sans risque pour les religions ${ }^{19}$. Ainsi la France initiée par Catherine de Médicis se poursuit-elle pour aboutir avec la Révolution. Au-delà de la rupture de 1789, la maxime cujus regio ejus religio reste toujours appliquée par la République, avec ce basculement vers une religion révolutionnaire. Le temporel continue à l'emporter sur le spirituel, dans cette ambiguïté d'une neutralité de l'État mâtinée de spiritualité politique. Désormais -sur les terres de la $1^{\text {èr }}$ République- on assiste plutôt au réveil de l'ancienne dispute entre État et Église, avec déjà l'annonce d'un accord impossible entre la nouveauté démocratique et la permanence des religions traditionnelles.

Depuis les grands débats révolutionnaires et pour tenter de définir, plusieurs versions du terme laïcité sont évoquées. Ces approches plurielles qui jaillissent dans les sens les plus contradictoires, ne cessent de s'entrechoquer. Elles chahutent la laïcité par des adjectifs qui la rendent équivoque, et la qualifient de positive ou de négative, de vraie ou de falsifiée, de fermée ou d'ouverte, de rigide ou de souple, de laïcité de dialogue ou de combat. Et l'on arrive même à parler bizarrement d'une laïcité laïque voire neutre ou au contraire idéologique et spirituelle ${ }^{20}$. Pour la France, cette dispersion signifie une ignorance complète du fait laïque qui est un signe grave de crise. 1994.

${ }^{19}$ Joseph Lecler, Histoire de la tolérance au siècle de la Réforme, Paris, Albin Michel,

${ }^{20}$ « Liberté d'expression, liberté religieuse en France, colloque international 10 et 11 avril 2014, Conflit des valeurs ? La liberté d'expression et la liberté religieuse, Université de Varsovie, à paraître. 
Chacun donne son avis. Comment alors expliquer l'attachement de l'opinion à la laïcité lorsque la laïcité elle-même est ignorée dans son histoire et son droit, lorsque sa définition est rendue peu sûre entre tolérance et intolérance à l'égard du fait religieux ? Émile Poulat n'hésite pas : « la laïcité, personne ne sait ce dont on parle $»^{21}$. Face aux désaccords et à la vaste nébuleuse des définitions, il peut sembler difficile d'affirmer que « la laïcité est depuis plus d'un siècle le pilier du pacte républicain, une référence commune, un cadre collectif $\rangle^{22}$. Les inversions terminologiques empêchent d'en faire cette référence stable, elles en font une notion flexible selon la formule célèbre du doyen Jean Carbonnier. Peut-être cette flexibilité se fait-elle indice d'un désarroi politique, face au nouveau paysage religieux qui s'établit en France, avec en arrière-plan la vieille idée que la postmodernité a conservé, celle d'un antagonisme irréductible entre espace démocratique et espace religieux qu'il faut désormais restreindre. Regardons alors :

\section{L'ESPACE PUBLIC ET LES MOUVEMENTS VERS LE PLURALISME RELIGIEUX : ENTRE HIER ET AUJOURD'HUI}

On se souvient qu'en 1999, dans son essai Un monde sans Dieu, Philippe Schwartz annonça la fin des religions comme la conséquence de l'accomplissement démocratique. L'idée n'était pas nouvelle. Elle appartenait au Siècle des lumières et surtout elle soutenait les visions politiques qui rendaient synonymes intégration et disparition. Le politique devait absorber le religieux jusqu'à le rendre invisible, jusqu'à perdre la notion du divin à remplacer par une spiritualité démocratique. En 2011, lors du colloque sur L'enseignement des religions, le cardinal Jean-Louis Tauran, président du Conseil pontifical pour le dialogue entre religions, affirmait que « les deux totalitarismes du siècle dernier avaient ainsi prophétisé définitivement la mort de Dieu, program-

${ }^{21}$ Émile Poulat, « Le code de la laïcité est insatisfaisant », La Croix, 1 novembre 2011 ; « Enquête sur la laïcité perdue », Nouvelle revue Certitudes, avril, mai, juin 2003, n 14.

${ }^{22}$ Discours prononcé pour l'installation de l'Observatoire de la laïcité, 8 avril 2013. 
mé la persécution des croyants et décrété l'exclusion de la religion de l'espace public $»^{23}$. Or, aujourd'hui cette sortie du religieux est contredite par le mouvement actuel vers un pluralisme religieux : « ce qui peut surprendre, ajoutait le cardinal Tauran, c'est qu'après la fin de l'unanimité culturelle, la mise en quarantaine du religieux dans la sphère privée et le délayage des valeurs et des modèles, la religion est devenue en quelques années un facteur capital dans la vie culturelle, politique, économique et même dans l'enseignement $\gg{ }^{24}$. Le désenchantement du monde par une dissolution du religieux n'aura donc pas lieu. Bien au contraire, se profile plutôt un triomphe des religions. François Jourdan l'écrit : « C'est définitif, nous sommes entrés dans l'ère interreligieuse $»^{25}$, même si parfois disparaît la pratique traditionnelle au profit de la consécration du plus incertain et individualiste believing without belonging.

Cette évolution vers la diversité religieuse travaille la laïcité, car elle contient des risques d'affrontement liés aux négations antérieures des religions. Elle s'accompagne de malaises sociaux qui fragilisent les politiques d'intégration, comme la visibilité des signes et des rites religieux. Il y a aujourd'hui -pour illustrer les agacements provoqués par la permanence du religieux- une accumulation de faits divers, entre autres celui de la gare de Villefranche-de-Rouergue. Il s'agit d'un accrochage révélateur, car relié à ce phénomène collectif de dissensions, avec la plainte formulée en décembre $2013^{26}$ par un voyageur contre

${ }^{23}$ S. Ém. le Cardinal Jean-Louis Tauran, « religions, croyants, société », dans Christine Mengès-Le Pape (sous la direction de), L'enseignement des religions, approches laïques et religieuses, Toulouse, Presses de 1'Université de Toulouse, 2010.

${ }^{24}$ Ibidem.

${ }^{25}$ François Jourdan, La Bible face au Coran, Paris, éditions de l'Euvre, 2008.

${ }^{26}$ Il s'agit de la plainte formulée en décembre 2013 par un usager de la SNCF contre la présence visible d'une crèche derrière un guichet de la gare de Villefranche-de-Rouergue ; cet usager a dit son mécontentement « de voir un signe religieux ostensible dans un lieu public ». Très vite l'histoire est devenue sérieuse, car la SNCF a envoyé une note aux cheminots qui ont alerté la presse, il y eut un article dans Centre-Presse, puis l'affaire a fait le tour des médias nationaux le Figaro, Le Nouvel Observateur, L'Express, Le Point et bien d'autres, même les journaux télévisés, ont donné leurs commentaires. Les cheminots avaient ainsi pris l'habitude depuis plus de 10 ans d'installer cette crèche. Et la dispute a dérangé la quiétude de la gare de Villefranche-de-Rouergue, même la quiétude nationale. La crèche fut alors temporairement dissimulée sous une bâche et, sur la vitre, une affichette délicieuse d'impertinence 
la présence visible d'une crèche de la nativité derrière un guichet. Et l'usager de dire son mécontentement « de voir un signe religieux ostensible dans un lieu public ». Très vite l'affaire prit une ampleur démesurée, avec une note de la SNCF adressée aux cheminots qui ont alerté la presse. La crèche fut alors dissimulée sous une bâche, et sur la vitre une affiche expliquait que : " suite à la plainte d'un client, nous ne pouvons laisser exposer la crèche. Crèche que la gare de Villefranche met en exposition depuis dix ans ! $»^{27}$. Dès lors les réactions sont allées dans toutes les directions. Elles ont montré la difficile appréhension des notions de laïcité, d'espace public, de signes religieux ostensibles, de neutralité à imposer à l'État ou aux particuliers, avec un avis prudent de l'Observatoire de la laïcité : « La neutralité s'applique, commentait Jean-Louis Bianco, seulement à l'État et aux bâtiments de la fonction publique, comme les mairies ou les écoles. Il n'y a pas d'impossibilité à installer une crèche dans une gare, car si l'entreprise est privée avec une mission de service public, le lieu de la gare est un espace public, un peu comme la rue ». Puis le président de l'Observatoire remarquait : « Il ne faudrait pas oublier une chose : la laïcité, ce n'est pas un inter-

expliquait que : «Suite à la plainte d'un client, nous ne pouvons laisser exposer la crèche. Crèche que la gare de Villefranche met en exposition depuis dix ans ! ». Les employés, attachés à la tradition, ont eu beaucoup de mal à comprendre la polémique. « Cela fait dix ans que nous avons l'habitude d'installer une crèche sur l'un de nos comptoirs. Nous ne voyons pas pourquoi cela changerait ! », déplorait l'un d'entre eux. Face au retentissement formidable de l'affaire, la direction de la SNCF a voulu dédramatiser. L'affichette a été retirée et la crèche est redevenue visible. Le directeur régional de la communication, Philippe Blanquart, a alors insisté sur la dimension locale, c'est-à-dire aveyronnaise de cet évènement devenu dangereusement national, il a aussi parlé du dialogue qui devait être suscité pour éduquer à la laïcité. « La crèche est un signe religieux mais, autour de Noël, expliqua-t-il, c'est aussi une tradition importante pour les agents, on trouve des crèches dans beaucoup d'espaces publics ", a-t-il observé, puis il fit une profession au principe de neutralité qui rendait sa vision très compliqué, il ajouta « à la SNCF, comme dans toutes les sociétés, des questions se posent, c>est pourquoi on a questionné notre direction de l'éthique ». Comme par anticipation, le comité d'éthique de la SNCF avait déjà émis en décembre, un avis réaffirmant les principes de laïcité et de neutralité de l'entreprise. L'Observatoire de la laïcité a même réagi -ce qui montre la charge symbolique de l'affaire de Villefranche de Rouergue- cette réaction alla dans le sens d'un apaisement, puisque Jean-Louis Bianco a marqué cette « démesure ridicule ». Pour le président de l'Observatoire de la laïcité, l'affaire était juridiquement claire : «Noël, c'est aussi une tradition, une histoire! ».

\section{${ }^{27}$ Ibidem.}


dit, mais une liberté au contraire, celle de pratiquer sa religion ». Cette déclaration va loin, elle peut même contenir une contradiction des positions législatives et jurisprudentielles, surtout en matière de signes religieux ostensibles ou de dimension manifestement excessive selon les lois de mars 2004 et d'octobre 2010, avec les voiles islamiques, les prières dans la rue, mais aussi ceux plus traditionnels pour la France des processions, des croix et autres emblèmes religieux souvent retirés des monuments publics; puis il y a la difficulté souvent soulevée des jours fériés. Dans l'affaire de la gare de Villefranche-de-Rouergue, c'est d'ailleurs la réplique d'un agent : « Et après, va-t-on supprimer les jours fériés religieux ? Il ne va pas rester grand-chose mis à part le 14 juillet... $\rangle^{28}$.

Si ces entassements de chicanes -et parfois leurs débordementssont nouveaux pour la République selon la loi de 1905, ils ne sont pas inédits. L'histoire de France ou d'ailleurs est remplie de cette alternance entre refus ou acceptation de la variété des identités religieuses et de leur manifestation. Pour l'Ancien Régime, on peut ici rappeler les nombreuses hésitations des derniers Valois et de la Régente contre Rome et face aux nouveautés de la Réforme. Ce sont également les rigueurs de la politique louis-quatorzienne. Puis il y a les excès des convulsionnaires jansénistes de Saint-Médard, Louis XV décida la fermeture du cimetière, et une affiche fut alors apposée, elle disait cette incompréhension populaire face aux interventions du roi justifiées par l'ordre public : « de par le roi, défense à Dieu de faire des miracles dans ce lieu $»^{29}$. Cette inscription n'est pas sans préfigurer tous les avis par la suite affichés, celui de la gare de Villefranche-de-Rouergue en est un écho. À travers la régulation des cultes, il y a toujours la recherche d'une unité à la fois temporelle et spirituelle qui concorde autour de la devise classique « un roi, une foi, une loi ». Au-delà, on y entrevoit une unité orientée vers un ordre universel, ce souhait illusoire de l'imperium mundi. Lors des triomphes successifs du pouvoir temporel et à travers des déclarations valables pour tous les lieux et tous les âges, l'histoire de France parcourt cette voie ouverte sur l'universel qui stigmatise

\footnotetext{
${ }^{28}$ Ibidem.

${ }^{29}$ Voltaire, Euvres complètes, Gotha, Charles-Guillaume Ettinger, 1786, t. XXXIX, p. 186.
} 
la diversité dans une forte confusion de l'unité et de l'uniformité. Dès lors, seules les ressemblances rassurent, les diversités doivent disparaître. C'est l'un des sens de l'inquiétude pour la solubilité des religions dans la France de la laïcité, et en particulier pour l'adaptation des cultes nouveaux et en particulier de l'islam qui ne conçoit pas la distinction du politique et du religieux. On retrouve une telle appréhension -dans Réformer l'Islam- d'Abdou Filali Ansary : « la laïcité serait strictement opposée à l'esprit de l'islam $»^{30}$.

Face aux nouveautés provenant du pluralisme, les tracas les plus récents poussent à un grand souci de convergence, peut-être parce qu'il est plus facile pour le repos public de concevoir une religion accordée par l'État ou un syncrétisme spirituel fondé sur le pilier du « très flou petit dénominateur commun " qui n'incite qu'à une curiosité frileuse, dans les ressemblances et non dans les différences. Il peut donc s'agir d'un mélange plutôt profane et ésotérique vers une morale laïque ou un syncrétisme religieux. On y aperçoit le symbole de la tour de Babel que les traités anciens ont souvent sublimé. Dans le Colloquium heptaplomeres et à travers la République de Venise, Jean Bodin décrit cet air livré à l'utopie d'une concorde universelle ${ }^{31}$. Or dans l'universalité de la plateforme réduite des valeurs, il y a un refus de l'altérité dans des peurs mutuelles inavouées à la fois par les États et les Églises, par ceux qui parfois s'ignorent. Et l'on se contente des accommodations qui masquent les erreurs historiques, théologiques et juridiques, dans un déni passionnel des diversités.

Aujourd'hui ces mêmes embarras traversent les politiques les plus récentes, ils ont suscité quelques expériences intéressantes de formation laïque proposées en particulier aux imams récemment entrés en France pour les sensibiliser à l'histoire française et à celle plus récente de la laïcité. Ce sont des certificats de « connaissance de la laïcité » ou des diplômes universitaires organisés par les universités publiques et les instituts catholiques, souvent à la demande de l'État, dans un partenariat qui a surpris. Des éditoriaux l'ont signalé avec des titres provoca-

\footnotetext{
${ }^{30}$ Cité par François Jourdan, La Bible face au Coran, op. cit.

${ }^{31}$ Jean Bodin, Colloquium heptaplomeres de abditis rerum sublimium arcanis, 1587, édition française, F. Berriot, Genève, Droz, 1984.
} 
teurs : « ces curés qui enseignent la laïcité », « ces imams formés par les curés $»^{32}$. De telles associations montrent l'urgence des tensions. Elles interrogent l'avenir de la laïcité dans des territoires tiraillés entre la neutralité inévitablement partiale du politique et la vitalité des mouvements religieux ${ }^{33}$. En évitant la séduction de la naïveté, ces nouvelles formations devraient offrir une possible pacification -contre les exclusions communautaires- par la voie traditionnelle des études et du savoir.

\section{RÉFÉRENCES}

Claude Durand-Prinborgne, La laïcité, éd. Dalloz, 2004.

Convictions philosophiques et religieuses et droits positifs, colloque national de Moncton, Bruxelles, éd. Bruylant, 2010.

Dominique Laszlo-Fenouillet, La conscience, Paris, LGDJ, 1993.

Laïcité et liberté religieuse. Recueil de textes et de jurisprudence, Paris, Les éditions des journaux officiels, 2011.

La laïcité, archives de philosophie du droit, Paris, éd Dalloz, mars 2005, t. 48.

Le pluralisme, archives de philosophie du droit, Paris, éd Dalloz, mars 2006, t. 49.

Liberté d'expression et liberté de communication, Les nouveaux cahiers $d u$ Conseil constitutionnel, Paris, éd. Dalloz, 2012, n³6.

Philippe Chiappini, Le droit et le sacré, Paris, éd. Dalloz, 2006.

Krzysztof Wojtyczek, "Les religions et le principe d'égalité », Revue européenne de droit public, 2005, $\mathrm{n}^{\circ} 1$.

RELIGIE W PRZESTRZENI PUBLICZNEJ FRANCJI, WCZORAJ I DZIŚ

\section{Streszczenie}

Pytania dotyczące obecności religii w przestrzeni publicznej Francji należą dziś do szczególnie aktualnych. Wobec europejskiego kryzysu imigracyjne-

${ }^{32}$ Cité par François Jourdan, La Bible face au Coran, op. cit..

${ }^{33}$ Krzysztof Wojtyczek, "Les religions et le principe d'égalité », Revue européenne de droit public, 2005, n 1, p. 125. 
go nie można dziś rezygnować z prób udzielenia odpowiedzi o adekwatność stosowanych dziś rozwiązań. Współcześnie realizowana we Francji koncepcja laickości wyjaśniana jest bowiem często z odwołaniem się do wymogów polityki integracji oraz potrzeb i nacisków różnych grup religijnych. Wobec dokonujących się zmian, postrzeganych często i nie bez podstaw jako zagrożenie dla porządku publicznego, należałoby rozpocząć od zrewidowania pojęć, często mylnie rozumianych, które od wejścia w życie ustawy z 9 grudnia 1905 roku określają obszary wytyczone dla religii. Dotycząca tych kwestii praca badawcza wymaga konfrontacji sytuacji historycznej ze współczesnością. Takie porównanie pozwala na uwypuklenie elementów stanowiących nowość, związanych ze zjawiskiem, które można określić jako wypaczanie świadomości społeczeństw świata zachodniego. W celu naświetlenia tej sytuacji należy wziąć również pod uwagę najistotniejsze różnice istniejące pomiędzy religiami, na których koncentrowali się twórcy unormowań z 1905 roku oraz tymi, które w Europie Zachodniej odgrywają dziś coraz większą rolę w konsekwencji ruchów migracyjnych. Należy przede wszystkim podkreślić małą adekwatność stosowania do nich tych samych reguł, które odnosi się do chrześcijaństwa. Przyjmują one bowiem często koncepcje teokratyczne i nie akceptują rozróżnienia sfery sacrum i profanum.

Ttumaczenie: Agnieszka Dębska

Słowa kluczowe: Laickość, sekularyzacja, przestrzeń publiczna, integracja, pluralizm religijny, wolność sumienia i wyznania, religia w przestrzeni publicznej

\section{RELIGIONS IN THE PUBLIC SPACE OF FRANCE, IN THE PAST AND TODAY}

\section{Summary}

Questions concerning the presence of religion in the public space of France are today particularly valid. The attempts to answer questions about the appropriateness of the current solutions cannot be given up in the face of European crisis concerning the issue of immigration. The concept of secularism currently implemented in France is in fact often explained with reference to the require- 
ments of integration policy and the needs and pressure from various religious groups. Regarding the current changes, seen often, and not without a reason, as a threat to public order, the discussion should start with the revision of concepts, often mistakenly understood, that have defined the areas of religion since the entry into force of the Act of 9 December 1905. The research of these issue requires a juxtaposition of the present and a historical situation. Such a comparison highlights any new elements that are associated with the phenomenon, which can be described as an act of distorting the consciousness of societies of the Western world. In the light of this situation the essential differences between religions should be also taken into account; those on which the creators of regulations from 1905 focused and those which in Western Europe today play an increasingly important role in the consequences of migration. The same rules that apply to Christianity cannot be applied to them because often they take on the theocratic concepts and do not accept the distinction between the sacred and the profane.

Ttumaczenie: Anna Sieradzka-Wawryszczuk

Key words: Secularism, secularization, public space, integration, religious pluralism, freedom of conscience and religion, religion in public space 